\title{
Feasibility of diffusion weighting with a local inside-out nonlinear gradient coil for prostate MRI
}

\author{
Enamul H. Bhuiyan ${ }^{1}$, Andrew Dewdney ${ }^{2}$, Jeffrey Weinreb ${ }^{1}$, Gigi \\ Galiana $^{1}$ \\ ${ }^{1}$ Department of Radiology and Biomedical Imaging, Yale School of Medicine, USA. \\ ${ }^{2}$ Siemens Healthcare, Erlangen, Germany.
}

Corresponding Author. email: gigi.galiana@yale.edu, Gigi Galiana, Associate Professor, Radiology and Biomedical Imaging, Yale University, School of Medicine, Phone: +1-(203) 785-5052

\begin{abstract}
Purpose: Prostate cancer remains the 2nd leading cancer killer of men, yet it is also a disease with a high rate of overtreatment. Diffusion weighted imaging (DWI) has shown promise as a reliable, grade-sensitive imaging method, but it is limited by low image quality. Currently, DWI image quality is directly related to low gradient amplitudes, since weak gradients must be compensated with long echo times.

Methods: We propose a new type of MRI accessory, an inside-out and nonlinear gradient, whose sole purpose is to deliver diffusion encoding to a region of interest. Performance was simulated in OPERA and the resulting fields were used to simulate DWI with two compartment and kurtosis models. Experiments with a nonlinear head gradient prove the accuracy of DWI and ADC maps diffusion encoded with nonlinear gradients.

Results: Simulations validated thermal and mechanical safety while showing a 5 to 10-fold increase in gradient strength over prostate. With these strengths, lesion CNR in ADC maps approximately doubled for a range of anatomical positions. Proof-ofprinciple experiments show that spatially varying b-values can be corrected for accurate DWI and ADC.
\end{abstract}

Conclusions: Dedicated nonlinear diffusion encoding hardware could improve prostate DWI.

Keywords: Diffusion weighted imaging (DWI), apparent diffusion coefficient (ADC), nonlinear gradient, prostate cancer, system design 


\section{Introduction}

\section{I.A. Prostate DWI}

The lifetime risk of being diagnosed with prostate cancer is approximately 1/9 for American men ${ }^{1-3}$, but because of slow progression and the significant morbidities associated with treatment, many men should consider declining, or at least delaying, treatment ${ }^{4,5}$. On the other hand, prostate cancer remains the $2^{\text {nd }}$ leading cancer killer of men (following only lung cancer), so early detection of aggressive cancers remains an urgent problem. Therefore, one of the greatest needs in prostate cancer is a monitoring method that distinguishes benign or low grade prostate lesions from aggressive ones. Standard template biopsy, which blindly samples $10^{+}$cores in a regular pattern, risks overdiagnosis, underdiagnosis, and urinary and erectile dysfunction, making it a poor option for diagnosis or surveillance ${ }^{6,7}$.

Diffusion-weighted MR imaging (DWI) does noninvasively detect prostate cancers ${ }^{8-10}$ and the images can be quantified into maps of apparent diffusion coefficient (ADC), which consistently reflect Gleason score and aggressiveness. Therefore, DWI has potential to specifically improve the detection of deadly cancers. Brightness in a DWI image (or darkness in an ADC map, with inverted contrast) reflects restricted water diffusion, so it is sensitive to the decreased luminal space and increased epithelial volume that accompanies malignancy. For men with high PSA (prostate specific antigen), prior MRI, typically can reduce unnecessary biopsies by a third ${ }^{11}$. In addition, these images can be used for biopsy guidance, increasing detection of high grade cancer and reducing biopsies of low grade disease ${ }^{12-15}$. However, clinical adoption has been limited by the poor image quality observed in routine prostate DWI, which is a key part of the multiparametric protocol. Fig. 1 shows typical image quality from a prostate imaging protocol. While the structural T2w image (Fig. 1a) has good SNR and resolution, it shows little contrast between cancerous and benign tissue. This is not uncommon for peripheral zone lesions, which make up $\approx 75 \%$ of cancer $\operatorname{cases}^{8}$. The ADC map (Fig. 1b), though very noisy and low resolution, does show markedly lower ADC in what was later confirmed to be an aggressive lesion. However, it was initially misread as artifact, due to the poor image quality. Images like these result in inconsistent interpretations, with high interreader variability, and limited clinical deployment ${ }^{16}$.

Last edited Date : November 25, 2020 
T2w Anatomical Reference

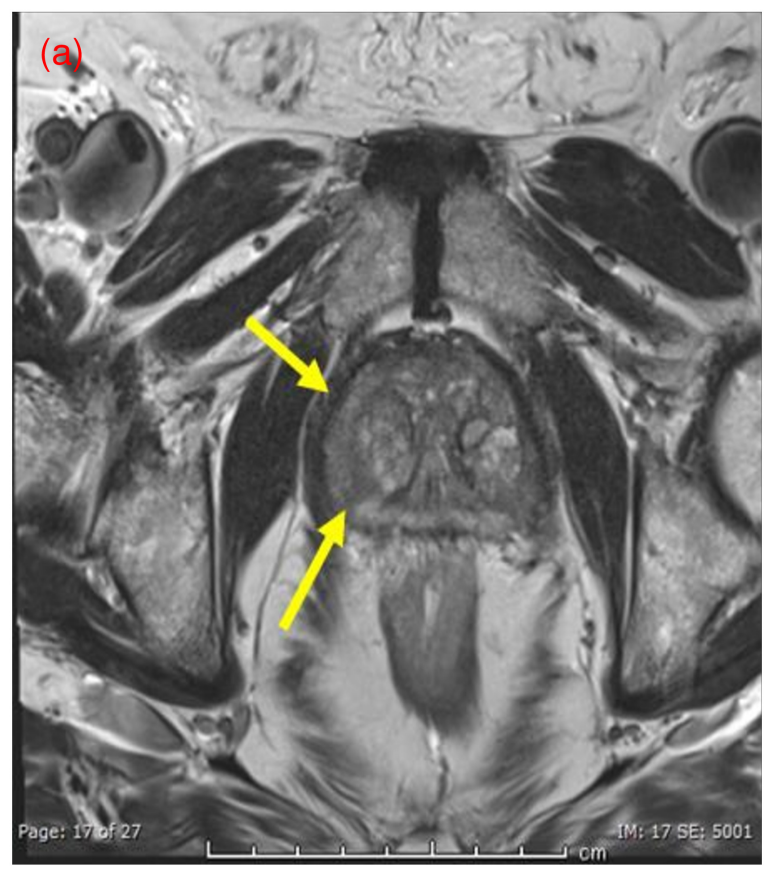

\section{Apparent Diffusion Coefficient (ADC) Map}

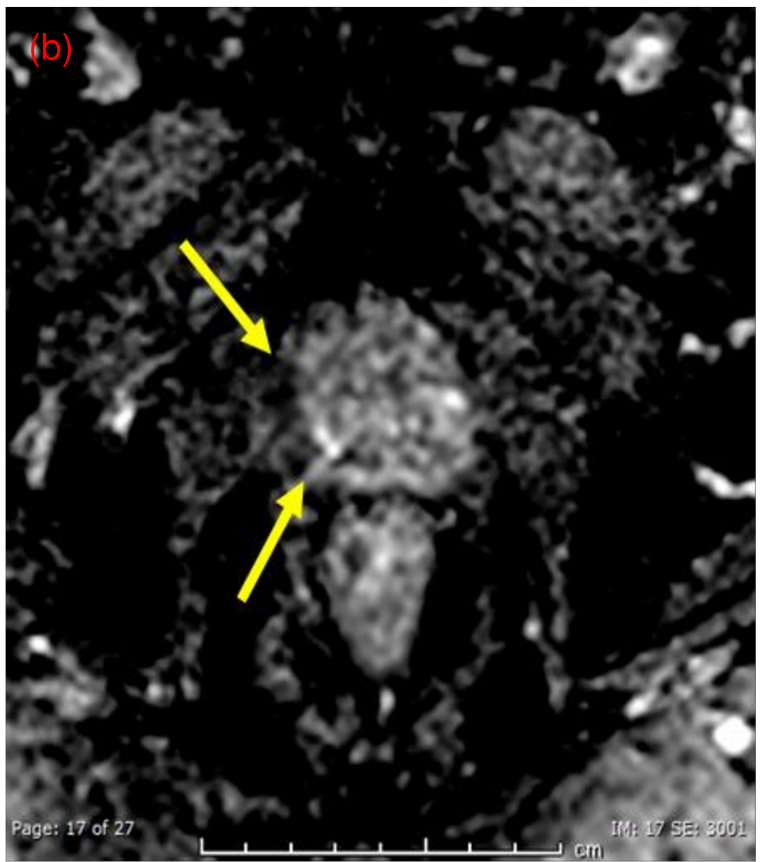

Figure 1: Images from a typical prostate MRI underscore the need for better imaging. (a) While a T2w image has high signal and resolution, it often fails to show contrast for peripheral zone tumors, which make up $\approx 75 \%$ of cases. (b) In the same patient, a hypointense region in the $\mathrm{ADC}$ map (i.e. a region of low diffusion) does distinguish the cancerous region from healthy prostate. But the overall image quality is low due to the low SNR of conventionally encoded diffusion MRI. Because of these shortcomings in prostate imaging, most clinicians rely on blind biopsies of the prostate, which can lead to over- and under-diagnosis of lethal disease.

Prostate DWI images are noisy because diffusion weighting depends exponentially on the square of gradient strength, and limited gradient strength must be compensated for by applying the gradient for very long times. The most widely used and simplistic model of diffusion is for Gaussian unrestricted diffusion, where:

$$
S_{D W I} \approx S_{0} e^{\left(-D \gamma^{2} G^{2} \delta^{2}\left(\Delta-\frac{\delta}{3}\right)\right)} \equiv S_{0} e^{(-D b)},
$$

where $S_{D W I}$ and $S_{0}$ are the signal intensities with and without diffusion weighting, respectively, $D$ is the intrinsic diffusion rate of the spins, analogous to the apparent diffusion coefficient (ADC) observed in vivo, $\gamma$ is the gyromagnetic ratio, $G$ is the local gradient 
amplitude, and $\Delta$ and $\delta$ are timings related to the duration of the gradient pulses which contribute to the overall echo time $(\mathrm{TE})^{17,18}$. The known experimental parameters in this expression are typically grouped together as a single measure of diffusion weighting, $b$. Using standard clinical gradients, strong diffusion weighting requires echo times of $\approx 100 \mathrm{~ms}$, during which signal decays precipitously. Furthermore, long TE overemphasizes the luminal volume of the prostate, which reduces contrast between aggressive and benign tissues. Empirical studies on the impact of TE and diffusion encoding time have shown that shorter encoding times and higher b-values increase the ability to distinguish between benign and cancerous lesions ${ }^{19-24}$. The need for gradients that can deliver strong diffusion weighting in short times motivated the search for alternative possibilities in gradient design.

\section{I.B. Nonlinear Gradients}

Existing gradient hardware generates a $B z$ magnetic field whose magnitude changes linearly with position (Fig. 2a, red line), so the local field slope or gradient is uniform across the field of view. Nonlinear gradients similarly generate a $B z$ whose magnitude varies in space (Fig. 2a, blue line), but the magnitude and direction of the field slope is different at different locations ${ }^{25-28}$. Put another way, for a given $B z$, linear gradients distribute gradient amplitude $(\|\nabla B z\|)$ with perfect equality to all positions in the field of view (FOV). (Fig. 2b) A comparable nonlinear gradient, by mathematical necessity, concentrates the gradient amplitude, leaving some regions with almost no gradient, but in other regions providing much higher gradient. For example, in the theoretical example of Fig. 2, a significant region in the field of view receives $>5$ times the gradient strength of the comparable linear gradient field.

This insight can be used to design hardware that efficiently generates very strong gradients in a particular anatomy of interest. For the targeted region of interest, the strong gradients deliver strong diffusion weighting in a short encoding time, thereby increasing signal to noise ratio (SNR) and contrast. On the scale of a voxel or the distance travelled by a diffusing spin, the field change remains approximately linear, so the same equations are applicable, except that gradient strength varies from voxel to voxel. This approximation has been confirmed by both experiments (Fig. 7 and Fig. 8) and first principles derivations (see 
E. H. Bhuiyan et al.
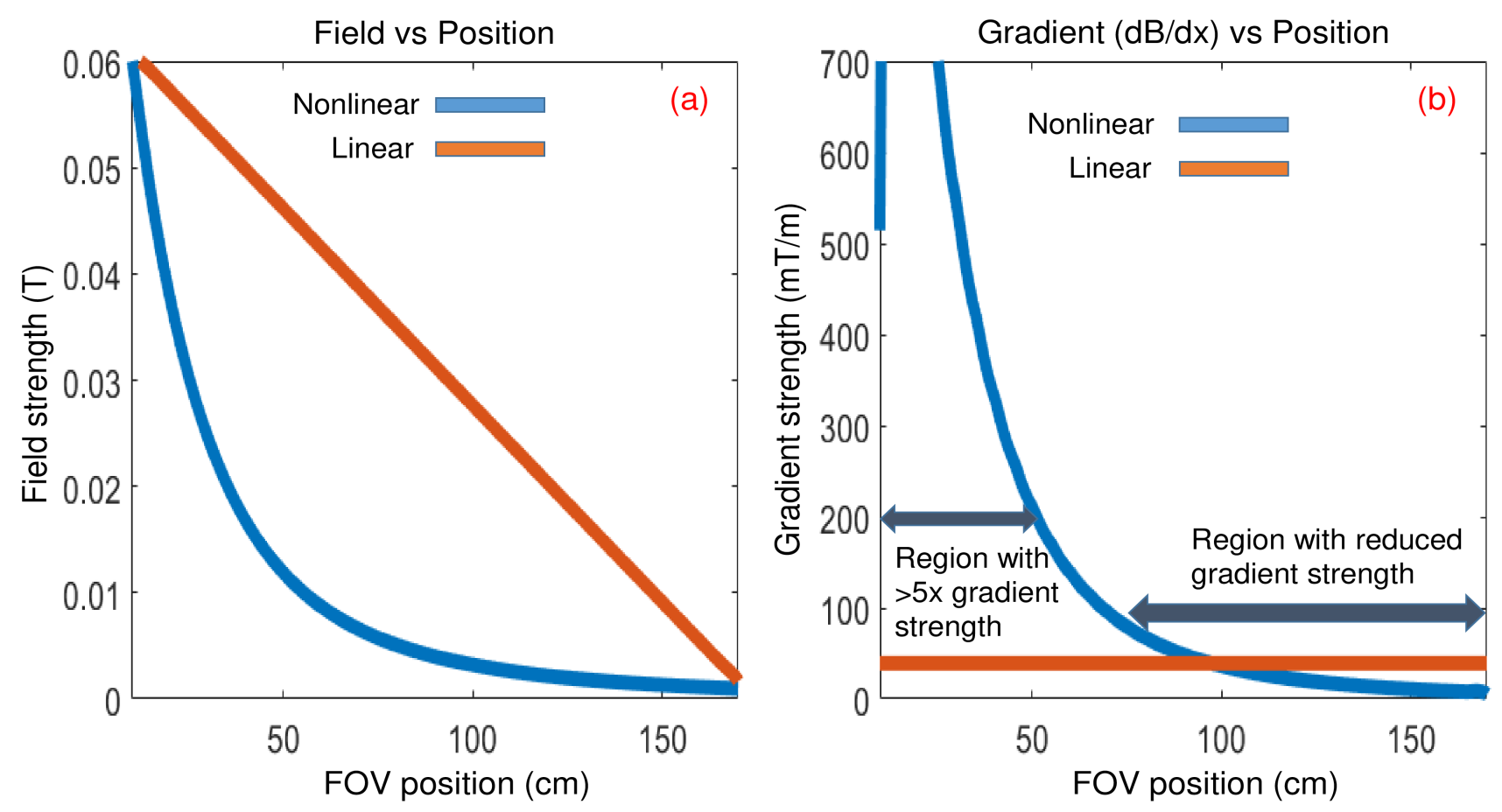

Figure 2: Conceptual schematic showing the inherent advantages of linear vs nonlinear field variations. For a maximum $\|B z\|$ of $60 \mathrm{mT}$ over this FOV (2a), linear gradients provide all points with the same gradient strength $(2 \mathrm{~b})$ of $36 \mathrm{mT} / \mathrm{m}$. A nonlinear gradient with the same maximum $\|B z\|$, by mathematical definition, provides less gradient in some regions but more gradient in others. For part of the field of view, this results in a much stronger gradient that can be used to impart diffusion weighting. In practice, the advantages of a nonlinear diffusion gradient are even greater, including fewer cancellations which would normally be needed to achieve linearity and potential for inside-out single-sided gradient designs.

\section{APPENDIX I).}

Designing a gradient specifically for diffusion weighting, rather than repurposing hardware primarily designed for spatial encoding, brings additional advantages for increasing gradient strength. The usual requirements on slope uniformity, both in magnitude and direction, require many field cancellations which ultimately waste amplifier energy. Reducing the volume over which the field must be generated may also reduce power needs ${ }^{29}$.

In fact, nonlinearity allows for hardware designs that do not encompass a volume at all, like the one presented here. Finally, since diffusion weighting requires just a few ramps per TR, short rise times are less critical. This allows higher inductance, more turns and therefore more field per amp. Alternatively, less voltage is required to achieve the slew rate, 
which reduces requirements on the amplifier and lowers the voltage breakdown strength requirements in the gradient coil ${ }^{29,30}$. Linear gradients, which can provide fast switching times and homogeneous spatial encoding over the entire FOV, can still be used for the rest of the pulse sequence, including slice selection, frequency and phase encoding, or any kind of preparation module. Therefore, standard DWI pulse sequences can be modified by simply adding a synchronization pulse that turns the nonlinear gradient on and off during diffusion encoding time. Notably, the nonlinear gradient can be played simultaneously with linear gradients, either for spatial encoding purposes like a prephaser, or to further add to the diffusion encoding gradient. When the spatial encoding is done by conventional means, the reconstruction is similarly conventional and can use regular FFT.

\section{I.C. Nonlinear Gradients for Prostate DWI}

Anatomically, DWI encoding with nonlinear gradients (NLG) is most naturally suited to an organ that is (i) small, so that each experiment yields an acceptable range of b-values across the region of interest (ROI), (ii) close to an accessible surface of the body, since gradients are typically strongest near the device, and (iii) structurally isotropic, so that variation in gradient direction is not a major confound. In practice, to justify additional hardware, the DWI contrast would also need to be a critical image for diagnosis. Thus, prostate cancer imaging is an ideal initial target for this type of hardware. The prostate is a small $4 \mathrm{~cm}$ organ located only $\approx 6 \mathrm{~cm}$ from the perineal surface with relatively isotropic diffusion, and prostate cancer is a widespread and deadly disease which is best imaged by DWI MRI. Furthermore, prostate MRI is a rapidly growing application, as it is increasingly recognized as a cost-effective step following elevated $\mathrm{PSA}^{31-37}$. Therefore, this was chosen for a first and ideal organ to benefit from this class of device.

Putting these insights together, we designed an inside-out gradient that provides very high diffusion weighting at the prostate. Standard gradient hardware is generally built into a volume-encompassing former, typically a tube-like shape, and the relevant fields are generated inside the volume of the hardware. In contrast, nonlinear gradients windings can be placed in a wand-type former, generating the relevant fields outside the hardware. The proposed device has a diameter of $10 \mathrm{~cm}$, comparable to a typical coffee mug, and can be placed between the upper thighs to achieve close proximity to the prostate. (Fig. 3) This 
E. H. Bhuiyan et al.

geometry also minimizes interference with the standard linear gradients, which can be used for spatial encoding in the usual way, and standard receiver geometry, which is typically a set of anteriorly and/or posteriorly positioned surface coils.
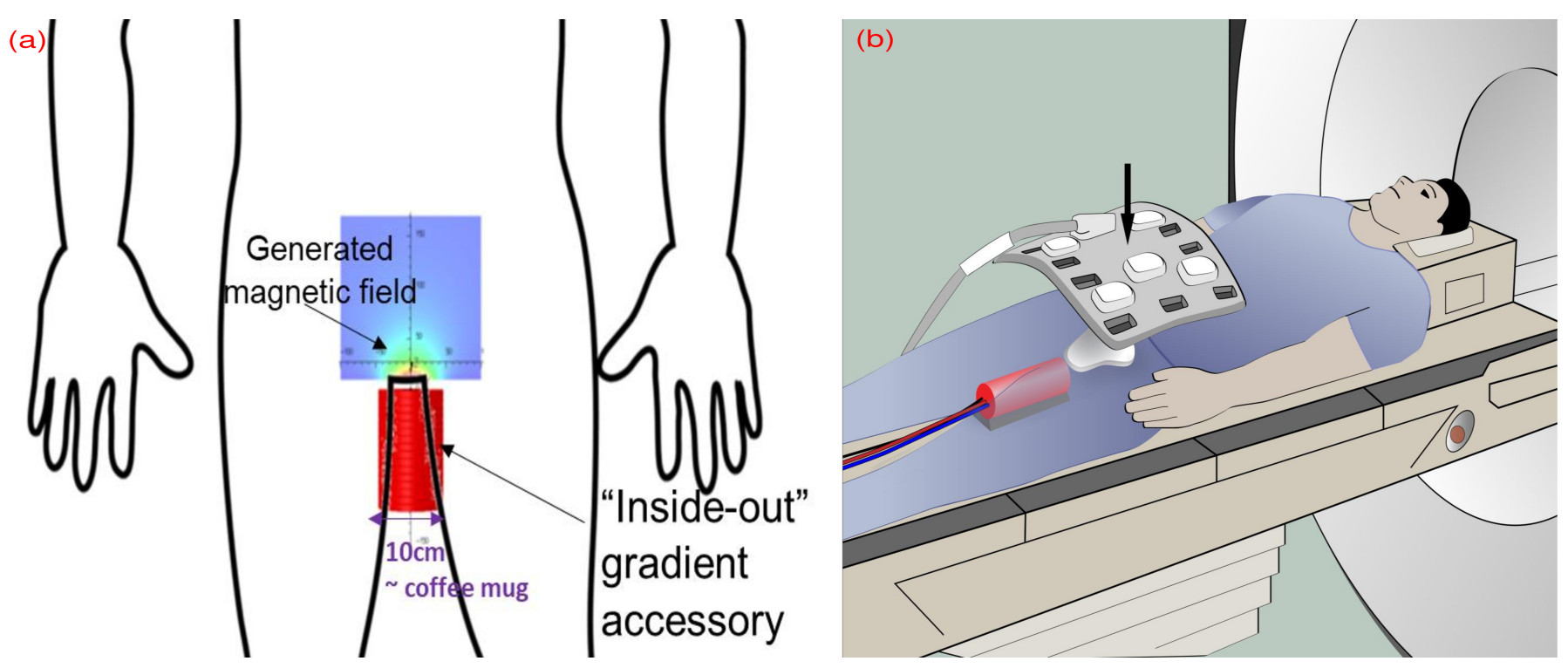

Figure 3: Positioning logistics for proposed hardware. Panel (a) shows general device placement and an overlay of the generated field. Panel (b) shows positioning in the scanner along with placement of a flex receive array. The gradient is placed externally, posterior to the genitalia and anterior to the anus, which provides close proximity to the prostate.

\section{Methods}

\section{II.A. Simulations}

From analyses of body MRI scans, the prostate is approximately a $4 \mathrm{~cm}$ sphere whose lower edge is $6 \mathrm{~cm}$ superior from the perineal surface. The presented prototype was designed by Tesla Engineering Ltd and simulated using OPERA Electromagnetic Simulation Software (Oxfordshire, UK). This design was then analyzed by Siemens Healthcare GmbH for wholebody forces and coupling effects with the Siemens superconducting magnet and gradient coils of a MAGNETOM Prisma with in-house software. All imaging simulations are performed in Matlab assuming a two compartment model comprised of a luminal compartment and a joint cellular and epithelial compartment. Parameters taken from ${ }^{20-23}$ were used to set:

\section{METHODS}


$\mathrm{T} 2_{l}=250 \mathrm{~ms}, \mathrm{~T} 2_{c}=50 \mathrm{~ms}, \mathrm{D}_{l}=2.5 \mu \mathrm{m}^{2} / \mathrm{ms}, \mathrm{D}_{c}=1.3 \mu \mathrm{m}^{2} / \mathrm{ms}$. Recent studies suggest the main driver of increased ADC with malignancy is loss of the luminal compartment volume, so this fraction was varied as 30\%, $15 \%$ and $7 \%$ for healthy prostate, medium grade prostate cancer in a lesion on the left, and aggressive prostate cancer in a lesion on the right, respectively ${ }^{24}$.

The multicompartment model introduced nonmonoexponential signal behavior observed clinically, both in the signal as a function of b and in ADC as a function of echo time. However, as done clinically, the resulting signal as a function of b was fit to a simple monoexponential to create maps of ADC. Since these experiments are meant to model the commonly used spin echo DWI sequence with $\Delta<100 \mathrm{~ms}$, rather than a STEAM sequence, the impact of $\Delta$ on $\mathrm{ADC}$ was neglected. While $80 \mathrm{mT} / \mathrm{m}$ gradients are now available commercially, these are often associated with very expensive and comprehensive upgrades. Thus we have performed comparisons against the more commonly available $40 \mathrm{mT} / \mathrm{m}$ gradient strengths.

Simulated ADC maps were generated using b-values of 60, 600, and 1500 for linear gradients and a maximum gradient strength of $40 \mathrm{mT} / \mathrm{m}$. For nonlinear gradients, diffusion gradient duration was chosen according to the prostate position in the FOV so that the least encoded pixel in prostate achieved a b value of 400. Diffusion weighted images were then simulated for 20\%, 45\%, 75\% and 100\% gradient strength. The highest b-values used for ADC calculation were 3000. Diffusion values higher than this were sometimes induced in the 4th DWI image for pixels very close to the device, but in this case the first 3 DWI images were sufficient for ADC calculation. Studies of position used a noise power of 15 .

Simulations were also performed for a model of diffusion which includes kurtosis, with $\mathrm{T} 2 / \mathrm{D} / \mathrm{K}$ equal to $130 \mathrm{~ms} / 1.89 \mu \mathrm{m}^{2} / \mathrm{ms} / 0.63$ and $80 \mathrm{~ms} / 1.41 \mu \mathrm{m}^{2} / \mathrm{ms} / 1.19$ for healthy and cancerous prostate tissue, respectively. In these simulations, images were simulated for gradient strengths of 20,30,40,55,70, 85, $100 \%$ maximum amplitude for both linear and nonlinear gradients, and noise power was reduced to 5 . Durations were set to achieve a maximum b-value of 3000 in the linear experiment and a minimum b-value of 500 in the nonlinear experiment.

Due to the spatially varying b-value in nonlinear experiments, the raw data from these scans also exhibits spatially varying contrast and spatially varying SNR. While the nonlinear scans can be used to make DWI with uniform b-value (as shown in Fig. 7), the fidelity of those images is ultimately determined by the fidelity of the ADC map used for scaling. Therefore, 
E. H. Bhuiyan et al.

as an equitable metric to compare nonlinear and linear experiments, we calculated CNR in $\mathrm{ADC}$ maps as: $\left(\mu\left(\mathrm{ADC}_{\text {lesion }}\right)-\mu\left(\mathrm{ADC}_{\text {normal }}\right) /\left(\sigma\left(\mathrm{ADC}_{\text {normal }}\right)^{2}+\sigma\left(\mathrm{ADC}_{\text {lesion }}\right)^{2}\right)^{1 / 2}\right.$, where $\mu$ is the mean and $\sigma$ is the standard deviation over each ROI. In simulations, since the exact delineation of healthy prostate and lesions were known, these were used as the ROIs for each tissue type.

\section{II.B. Experiments}

To prove the feasibility of encoding diffusion with a nonlinear gradient, initial experiments were performed with an existing nonlinear gradient, a volume encompassing head coil that produces a C3 field shape $B z(x, y)=x^{3}-3 x y^{2}$. These studies imaged a kiwi, which has been lauded as a highly concordant phantom for prostate DWI ${ }^{38}$, embedded in gelatin and a nominal gradient strength of 816,1632 , and $2449 \mathrm{mT} / \mathrm{m}^{3}$ with $\mathrm{TE}=32 \mathrm{~ms}$ and gradient duration of $12 \mathrm{~ms}$. The linear experiments on this phantom used strengths of 10, 20, and $30 \mathrm{mT} / \mathrm{m}$ with $\mathrm{TE}=60 \mathrm{~ms}$ and gradient durations of $26 \mathrm{~ms}$. All experiments were acquired with a 1-channel $\mathrm{Tx} / \mathrm{Rx}$ head coil nested inside the existing nonlinear gradient insert. In nonlinear gradient studies, scaling to a uniform b-value was performed by using raw data with acquired b-values to generate an ADC map and then extrapolating, via a monoexponential fit, to the desired b-value ${ }^{39}$.

Additional experiments were performed on a High Precision Device (HPD) diffusion phantom Model 128 containing thirteen $30 \mathrm{ml}$ vials of the nontoxic polymer polyvinylpirrolidone (PVP) in aqueous solution having different concentrations of $\mathrm{PVP}^{40,41}$. As the ADC depends on temperature, it is necessary to maintain a constant temperature in order to achieve reproducible ADCs. Coexistence of ice and water in the phantom serves to maintain temperature at $0^{\circ} \mathrm{C}$. Because b-values from NLG encoding vary substantially over this relatively large phantom, gradient durations that achieved reasonable diffusion weighting for one vial would provide too much or too little diffusion weighting in another vial. Furthermore, regions far from the bore periphery have very little diffusion weighting even at long gradient durations. Instead, across a series of studies with increasing gradient duration, we identified, for each vial, the study where the maximum b-value averaged across the ROI was closest to a target value of 1500. ADC for the region was then calculated using that experiment and compared to the result from a conventional DWI experiment using linear gradients. Linear 
experiments on this phantom used TE $=61 \mathrm{~ms}$, gradient lobes of $26 \mathrm{~ms}$, and gradient strengths as in the kiwi experiment. NLG experiments used TE $=26-98 \mathrm{~ms}$ with gradient durations of 14-38ms and gradient strengths as in the kiwi experiment.

Field mapping was performed by incrementing the duration of a single gradient lobe (1.5-2ms) at low amplitude to avoid intravoxel dephasing, holding TE fixed at 20ms. The observed phased progression was used to calculate local frequency as $\omega(x, y)=\Delta \Phi(x, y) / \Delta t$, and this was used to generate a map of the static field, which was then masked and fit to ta polynomial to generate a field map across the entire FOV. The local gradient was calculated

as $\left(G_{x}(x, y)^{2}+G_{y}(x, y)^{2}\right)^{\frac{1}{2}}$, and this was used to calculate the b-values for each voxel. To control for any differences in processing, linear gradients and b-values were calculated by the same procedure, but high uniformity and agreement with the nominal field was observed, as expected.

For imaging in these proof-of-principle experiments, a 2D GRE sequence with bipolar diffusion encoding gradients was used with the following parameters, FOV $=250 \times 250 \mathrm{~mm}^{2}$, matrix size $256 \times 256, \mathrm{TR}=500 \mathrm{~ms}, 500 \mathrm{~Hz} /$ pixel receiver $\mathrm{BW}$, flip angle $50^{\circ}$. All experiments used standard gradient echo sequences with Cartesian trajectories played out on linear gradients and bipolar diffusion encoding gradients. The only difference between experiments was whether the diffusion encoding pulses were delivered on the linear vs nonlinear gradient hardware. All experiments were performed on a 3T Siemens Trio system.

\section{Results}

\section{III.A. Simulation of Hardware Performance}

Fig. 3a shows the general positioning and the field generated by the device, and Fig. 3b shows a mock-up of the device placed on the scanner bed. A patient would be positioned with the device externally nestled between the legs, and a receiver coil would be placed above these. Water and power cables would extend in the foot direction.

Simulations were also performed on this design to assess thermal and mechanical properties. Because the device is designed for placement at isocenter in the homogeneous region of the magnet, net forces due to device operation are expected to be negligible. Even for 
misplacement up to $\pm 1 \mathrm{~cm}$, forces are less than $1 \mathrm{~N}$ due to symmetry. Even in a maximally catastrophic case of complete symmetry loss at $100 \%$ current, forces on the device reach $<500 \mathrm{~N}$, which can be restrained with adequately designed scaffolding. Improper positioning could also affect image quality through coupling to the linear gradients, but preliminary analysis indicates that a positioning tolerance of $\pm 2 \mathrm{~mm}$ is both sufficient and achievable. The device is cooled and insulated such that surface temperature at maximum duty cycle will not exceed $42^{\circ} \mathrm{C}$. Other key metrics are reported in Table 1.

\begin{tabular}{|c|c|}
\hline \multicolumn{2}{|c|}{ Gradient Specifications } \\
\hline Diameter & $94 \mathrm{~mm}$ \\
\hline Length of active section & $110 \mathrm{~mm}$ \\
\hline Total length & $170 \mathrm{~mm}$ \\
\hline Resistance & $37 \mu \Omega$ \\
\hline Inductance & $305 \mu \mathrm{H}$ \\
\hline Peak Current & $625 \mathrm{~A}$ \\
\hline Peak Voltage & $500 \mathrm{~V}$ \\
\hline Steady state DC current & $550 \mathrm{~A}$ \\
\hline Dissipation at max DC current & $13 \mathrm{~kW}$ \\
\hline Pressure Drop & $0.68 \mathrm{bar}$ \\
\hline Parallel water circuits & 8 \\
\hline Flow rate & $8.8 \mathrm{~L} / \mathrm{min}$ \\
\hline Water inlet temp & $20^{\circ} \mathrm{C}$ \\
\hline Maximum temperature & $42^{\circ} \mathrm{C}$ \\
\hline
\end{tabular}

Table 1: Summery of the key metrics

Fig. 4 shows the generated field predicted for this design for a coronal plane assuming a $625 \mathrm{~A} 500 \mathrm{~V}$ amplifier, showing both the z-component of the gradient (4a) and the component in the transverse plane (4b). The device and field exhibit cylindrical symmetry about the z-axis. The typical expected position of a prostate is outlined in red. Putting these together as $\mathrm{G}_{t o t}(4 \mathrm{c})$, it can be seen that the total gradient magnitude varies mostly as a function of $\mathrm{z}$ (i.e., the head-foot direction). Therefore, gradient is plotted as a function of $\mathrm{z}$ in $4 \mathrm{~d}$, focusing on the ROI of a typical prostate. Fig. 4e shows a contour plot of b-values for a $1 \mathrm{~ms}$ pulse at full current. The overall topology of this plot is that of $\mathrm{G}_{t o t}^{2}$, and the values scale uniformly with the square of amplifier power. Minimum rise times to maximum current are 

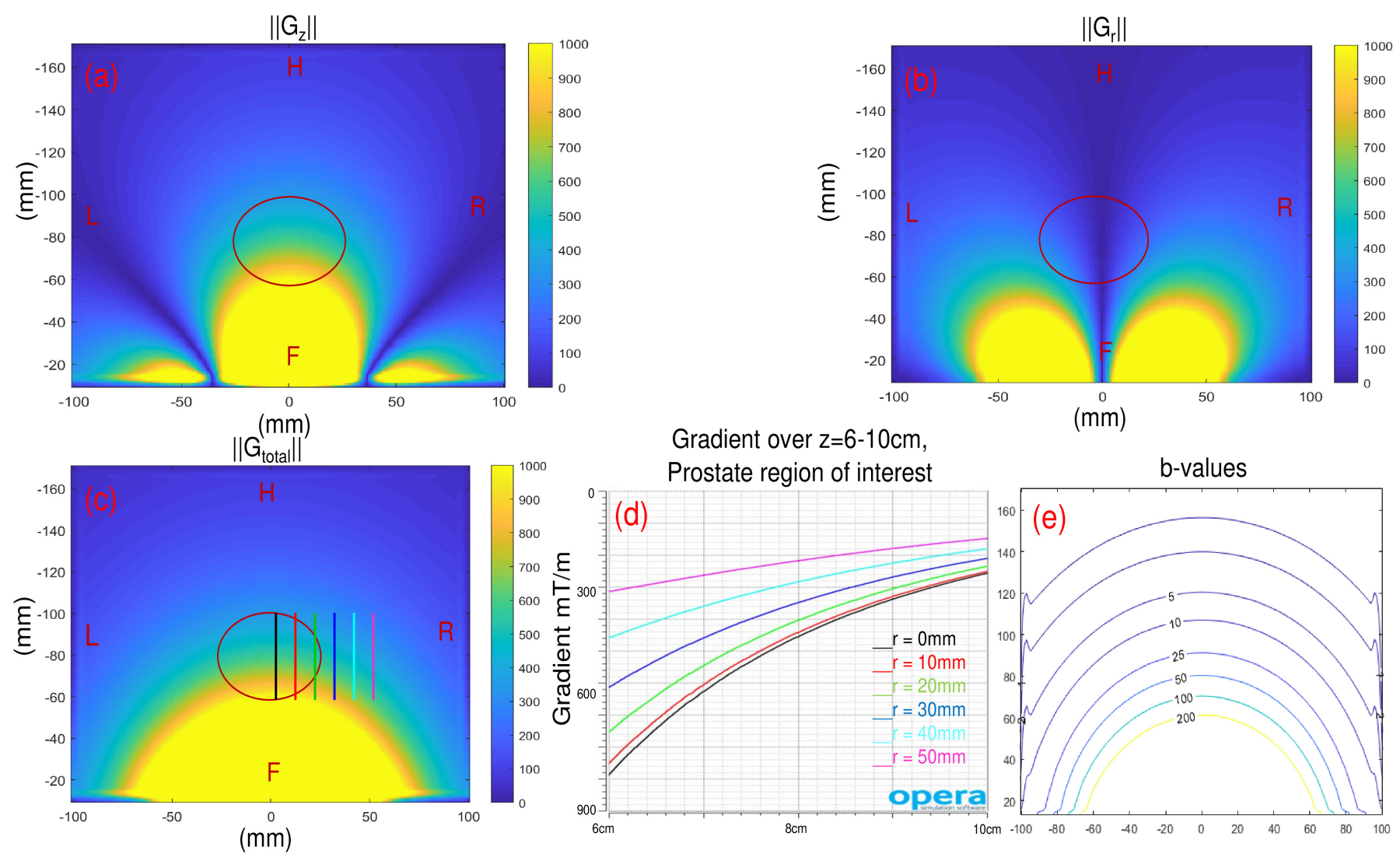

Gradient over $z=6-10 \mathrm{~cm}$, Prostate region of interest

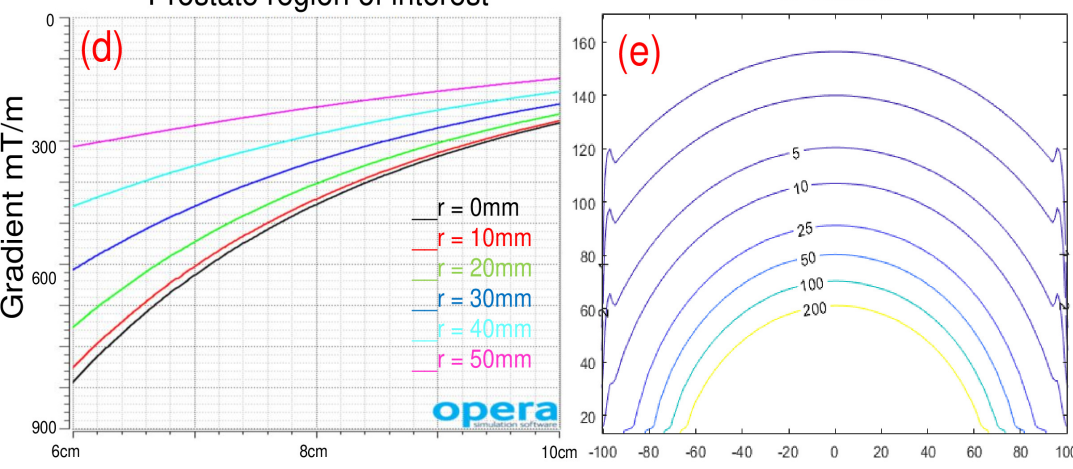

Figure 4: Images of gradient strength across the field of view. (a,b) Field variations in both the head-foot (a) and left-right direction (b) contribute to diffusion encoding. Summing these, the overall gradient magnitude (c) varies primarily along the head-foot direction. In each figure, the red outline shows the typical position of the prostate at closest approach. Various traces in the total gradient and a map of b-values are plotted in (d) and (e), respectively.

$397 \mu$ s, though practical rise times would depend on peripheral nerve stimulation, as further considered in Discussion.

\section{III.B. Simulation of ADC Map Improvement}

Fig. 5 shows simulations of the potential image improvements from implementing such a gradient. Because SNR and b-weighting vary spatially in images acquired with nonlinear gradients, standard DWI images would require scaling via an ADC map, so analyses focus on these. 


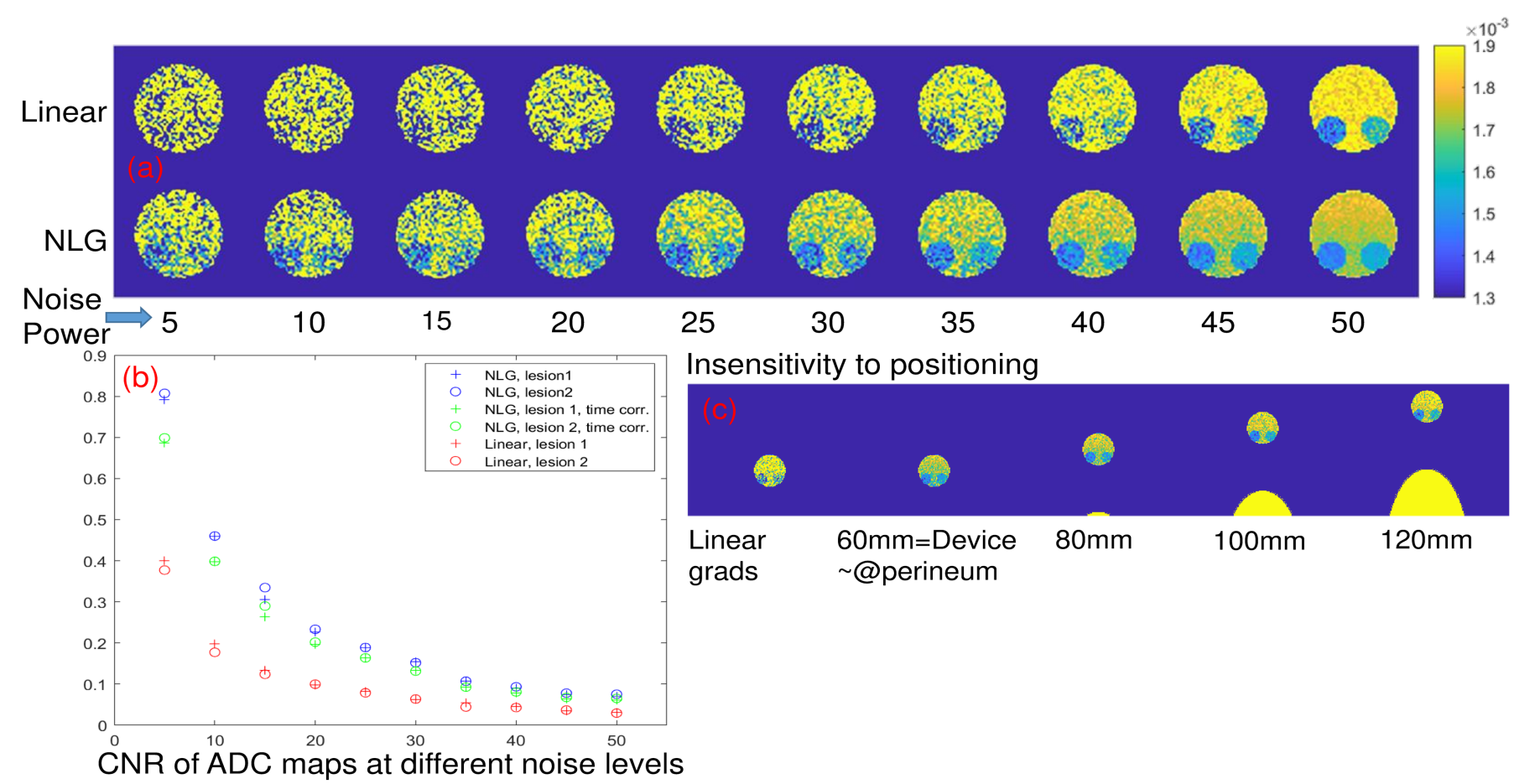

Figure 5: CNR of ADC maps simulated for diffusion encoding with linear and nonlinear gradients. (a,b) As expected, at the lowest noise levels, lesions are identifiable even with the longer echo times needed by linear gradients. However, the shorter echo time used with nonlinear gradients ultimately results in a consistent doubling of CNR in ADC maps. (c) With nonlinear gradients, position of the prostate relative to the hardware could potentially affect performance, as a longer diffusion time is required to achieve sufficient weighting. However, simulations show that this dependence is relatively minor, and CNR remains well above that observed with linear gradients.

\begin{tabular}{|c|c|c|c|c|c|}
\hline & Linear gradient & \multicolumn{4}{|c|}{ Nonlinear Gradient } \\
\hline Position (mm) & 60 & 60 & 80 & 100 & 120 \\
\hline CNR, Lesion 1 & $100 \%$ (ref) & $200 \%$ & $230 \%$ & $210 \%$ & $190 \%$ \\
\hline CNR, Lesion 2 & $100 \%$ (ref) & $200 \%$ & $230 \%$ & $210 \%$ & $190 \%$ \\
\hline Time corr. CNR Lesion 1 & $100 \%$ (ref) & $175 \%$ & $200 \%$ & $180 \%$ & $165 \%$ \\
\hline Time corr. CNR Lesion 2 & $100 \%$ (ref) & $175 \%$ & $200 \%$ & $180 \%$ & $165 \%$ \\
\hline
\end{tabular}

Table 2: CNR insensitivity to position

Fig. 5a shows simulated ADC maps generated with linear and nonlinear gradients at various noise levels. Fig. 5b, summarizes the contrast to noise ratios observed in each image for the medium and high grade lesions. The proposed nonlinear gradient is associated with 
a consistent doubling of contrast to noise over the standard method.

Fig. 5c simulates the effect of different positionings of the nonlinear gradient coil, mimicking either alternate placement or anatomy. The CNR improvements for these scenarios are summarized in Table 2. While these studies do reveal a weak dependence of CNR on position of approximately $\pm 10 \%$, all values are approximately a 2 -fold improvement over the contrast to noise ratio (CNR) achieved with linear gradients.

Fig. 6 explores the importance of these stronger gradients for higher b-value imaging with greater SNR to fit kurtosis in a higher order model of diffusion ${ }^{42,43}$. For the high SNR simulated in these experiments, the ADC maps are comparable in both cases. However, the kurtosis maps show much better delineation of the lesions when using nonlinear gradients.

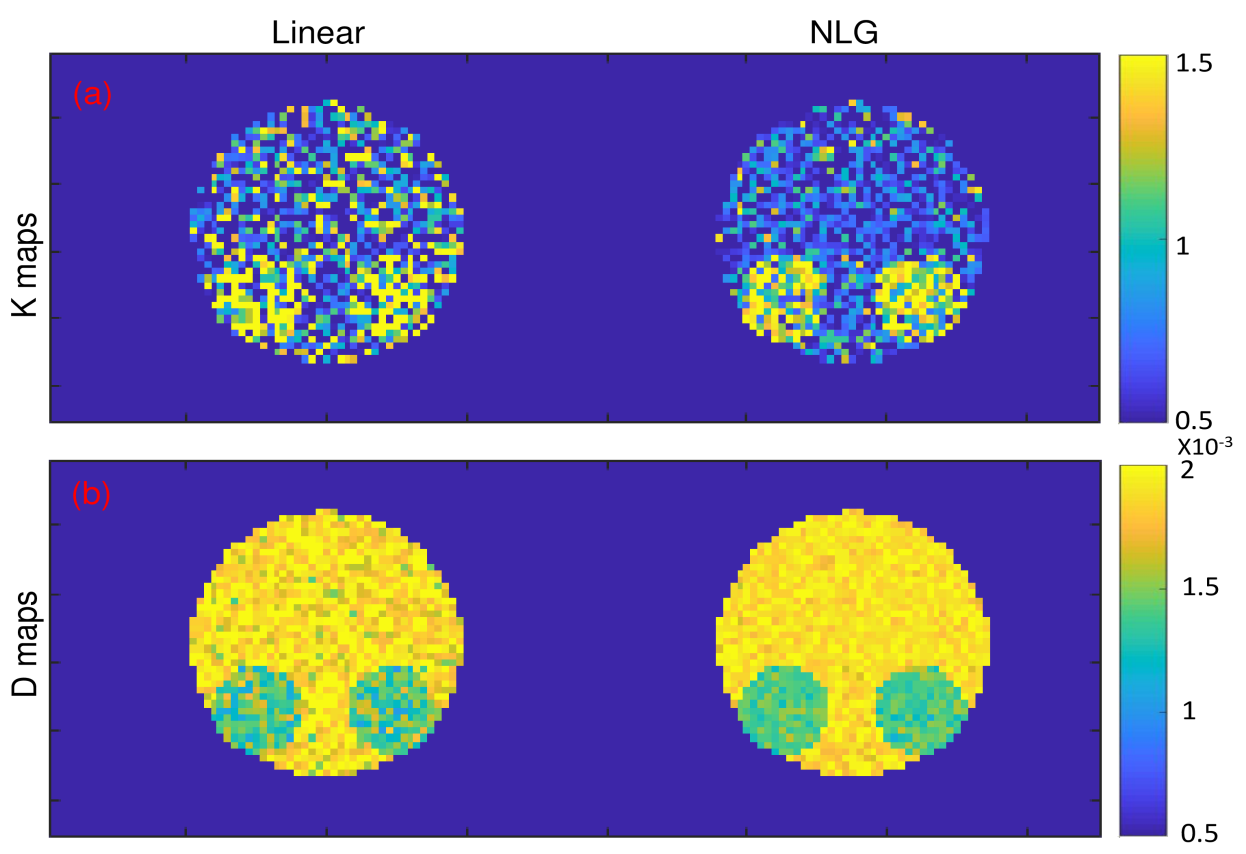

Figure 6: Fitting higher order models of diffusion. In this example, high b-value data was simulated with an additional second order term in the exponent of the standard diffusion model. The fit coefficient on this second order term is shown in (a), showing clearer delineation of the lesions with the NLG hardware. In these relatively low noise simulations, both ADC maps clearly delineate the lesion. 
E. H. Bhuiyan et al.

\section{III.C. Experimental Demonstration of DWI with NLG}

One key to achieving an order of magnitude increase in gradient strength is accepting nonlinearity in the field, which introduces nonuniform diffusion weighting across the image and could theoretically introduce bias in an ADC map. Thus we sought to show initial feasibility of DWI with nonlinear gradients using nonlinear gradient hardware available at our cen$\operatorname{ter}^{44-48}$. Though the geometry of this device is not suitable for prostate imaging, the degree of nonlinearity is comparable and these results provide proof of principle demonstrations for encoding diffusion with a nonlinear field.

Fig. 7 shows individual diffusion weighted images acquired with nonlinear (7a) and linear (7b) gradients. To generate the images in $7 \mathrm{a}$, the raw images must be scaled to a uniform b-value in the same way that low b-value images are routinely scaled to higher b-value images in clinical practice ${ }^{39}$. The raw images acquired with nonlinear gradients (7c) exhibit the spatial nonuniformity in diffusion weighting (blue arrows). As can be seen by comparing $7 \mathrm{a}$ to $7 \mathrm{c}$, standard b-value scaling corrects this variability while maintaining improved SNR from the reduced TE. Parameter maps generated from fitting the data to a monoexponential signal model show good agreement in the ADC maps $(7 \mathrm{~d}, \mathrm{e})$. The fit to $S_{0}$ $(7 f, g)$ reflects the much higher signal achieved in the nonlinear gradient study. Though these proof-of-concept experiments do not use gradients optimized for this sample or positioning and employ $\approx$ half the amplifier current, DWI with nonlinear gradients required less encoding time for comparable diffusion weighting across this phantom. This led to a doubling in signal, which is reflected in the maps of $S_{0}$.

To further explore the importance of the additional SNR associated with shorter echo times, the ROIs shown in panel (h) were used to assess image homogeneity over presumably homogeneous regions in both ADC maps and DWI images. These results, shown in panels (i) and (j) for agar and kiwi regions, respectively, do suggest improved precision in the images acquired with nonlinear gradients.

Finally, to probe quantitative ADC agreement, experiments were performed on the High-Precision Device (HPD) diffusion phantom shown in Fig. 8a. A summary of those results is shown in Fig. 8b. For vials 10 and 11, even with a gradient duration of $42 \mathrm{~ms}$ 


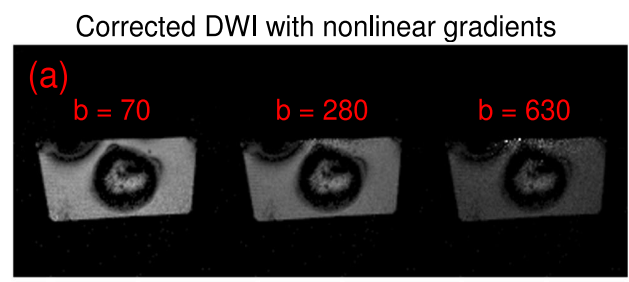

DWI with linear gradients

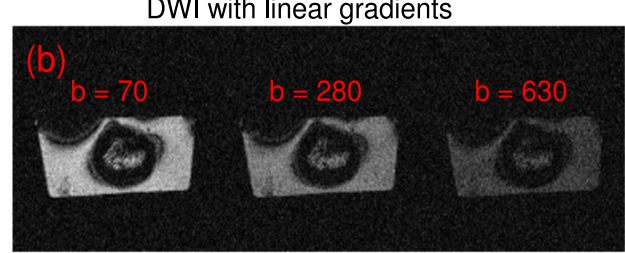

Raw images with nonlinear gradients
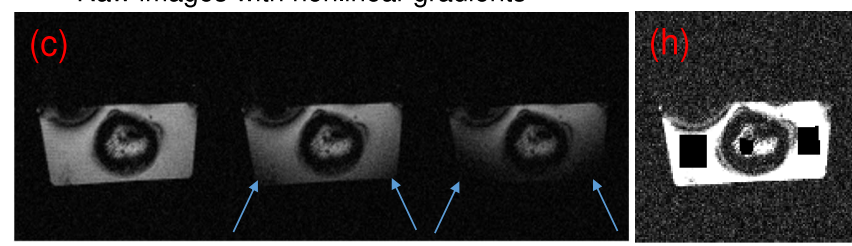

Note: Grayscale NLG and linear DWI images were scaled by 0.6 to compensate for the signal differences resulting from shorter echo times.
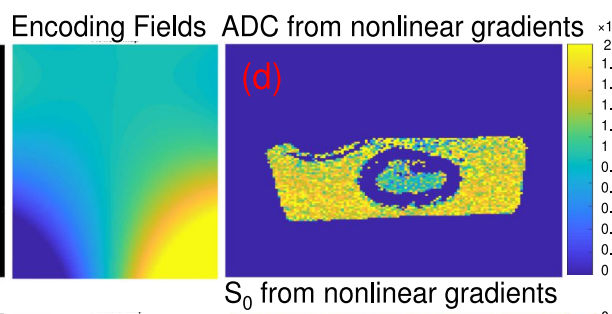

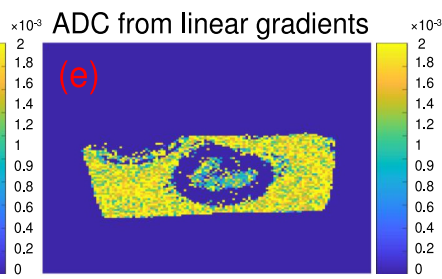

$\mathrm{S}_{0}$ from linear gradients

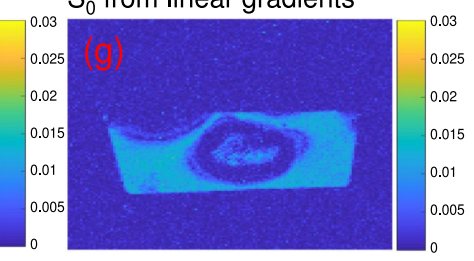

Comparison of signals in an agar ROI
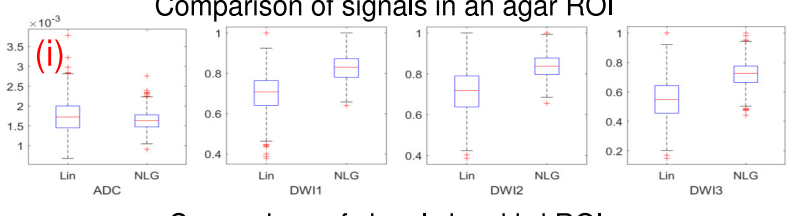

Comparison of signals in a kiwi ROI

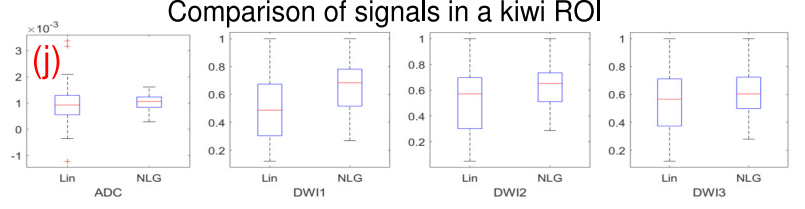

Figure 7: Experimental results from DWI with nonlinear and linear gradients. Panel (a) shows DWI images using a nonlinear C3 gradient and scaled to a uniform b-value. Standard images acquired by using linear gradients at these b-values (b) are shown for comparison and show excellent agreement. In the raw NLG images (c), the nonuniform diffusion weighting can be observed in the corners highlighted by blue arrows. Fitting this data to a monoexponential model yields ADC maps (d) that are in good agreement with those from linear gradients (e). Mapping $S_{0}$ from these monoexponential fits (f,g) shows that the shorter echo time afforded by nonlinear gradients results in about a doubling of signal. Furthermore, a comparison of image uniformity over the ROIs shown in (h) suggests the higher SNR of nonlinear DWI translates to better precision $(\mathrm{i}, \mathrm{j})$.

( $\mathrm{TE}=98)$, the maximum b-values were less than $200 \mathrm{~s} / \mathrm{mm}^{2}$, so ADC could not be accurately calculated. However, for the remaining vials, ADC agreement was very high. In these studies, because of the phantom geometry and the shape of the field, most of the vials required longer gradient durations for nonlinear gradient experiments. Thus, SNR of the underlying images and accuracy of the measured ADC was not necessarily better. However, these results provide reassurance that the diffusion contrast from nonlinear gradients closely matches that of linear gradients. 


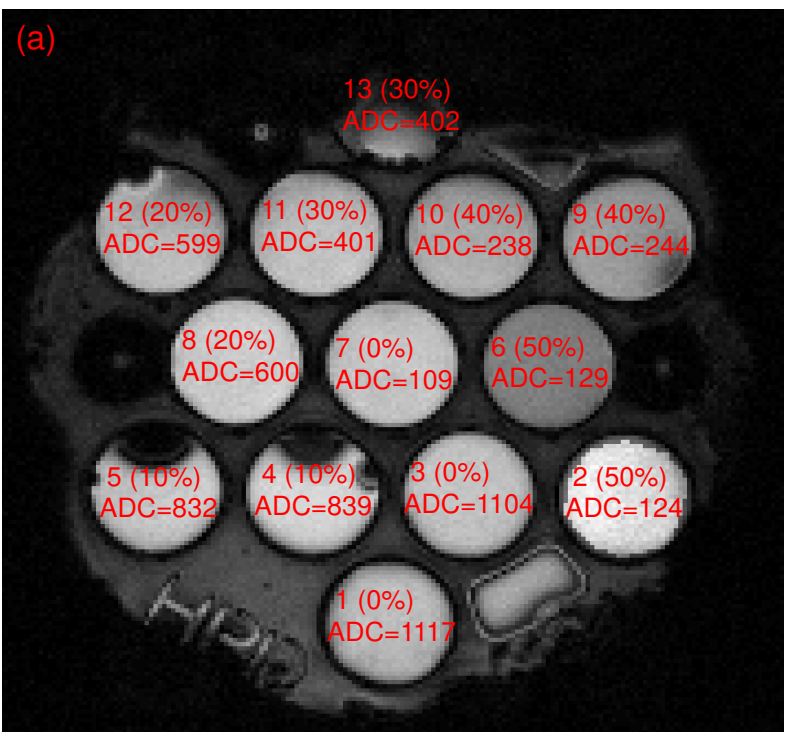

Vials and PVP concentration
Measured ADC vs Spec ADC

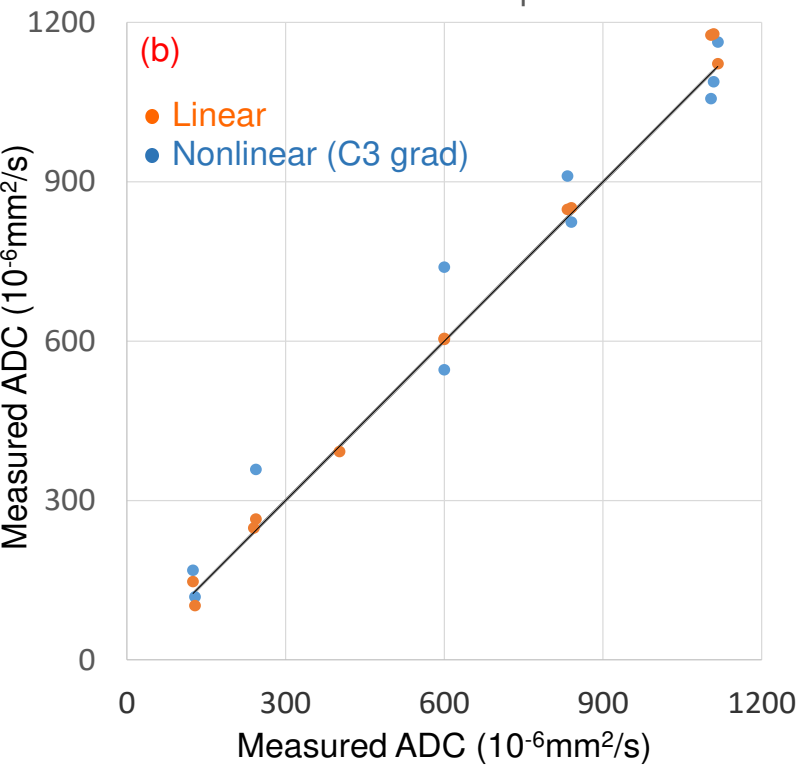

Figure 8: Quantitative comparisons of ADC in a diffusion phantom. (a) A diffusion phantom composed of vials of aqueous PVP (concentration in \%) was used to test the agreement between ADC values measured with linear and nonlinear gradients. The three smaller $5 \mathrm{~mL}$ vials are fiducial markers. (b) A comparison among measured ADCs shows good agreement.

\section{Discussion}

Because the device is meant only to deliver diffusion weighting, image encoding and reconstruction are unaffected. By focusing on $\|\nabla B z\|$ over the anatomy of interest, the concept presented here could be comparable in price to other anatomically specific MRI accessories, such as RF coils, and be portable between scanners. Yet this accessory would deliver an order of magnitude increase in gradient strength and double contrast to noise ratios in prostate cancer detection.

The presented design shows that by abandoning design specifications required for imaging, particularly unidirectional linearity of the gradient and cylindrical geometry, the local magnitude and diffusion encoding can be greatly improved. Recently, this potential has also been explored in a volume-encompassing design for breast imaging ${ }^{49}$. Our experimental results show that nonlinear gradients can produce diffusion weighting that is qualitatively and quantitatively comparable to standard methods, even for phantoms whose geometry is 
not particularly suited to the gradient topology. While current results focus on improved ADC mapping, more sophisticated models of diffusion could also potentially be probed using these much stronger imaging gradients ${ }^{21-24,50-56}$.

Several features make this approach highly feasible with minimal changes to an existing MRI system. Because diffusion waveforms for prostate DWI do not require a high degree of flexibility, a dedicated DWI gradient would only require the addition of pulse sequence synchronization pulses which then trigger a very simple waveform generator. Furthermore, while initial implementations will feed these waveforms to a dedicated amplifier, a DWI gradient of this kind could share the amplifier of one of the linear gradients via a switch. In either case, the rest of the pulse sequence, including excitation with the body coil, image encoding with linear gradients, reception with standard RF arrays, and Fourier transform image reconstruction, proceeds unmodified. During the diffusion encoding module, the linear gradients could either be turned off or run in tandem, to achieve still higher local gradient or effectively move the region targeted for optimal diffusion weighting.

One concern with nonlinear gradients is the sensitivity of CNR to position. If the prostate is too close to the device, there is higher gradient variability and additional DWI images might be needed to get adequate diffusion encoding across the entire prostate. Alternately, if the prostate is too far from the device, there may be concern that the gradient is too weak to yield a significant benefit. While more sophisticated algorithms are possible for optimizing gradient durations and amplitudes, the presented simulations show that even a rudimentary algorithm for modifying $\Delta$ as a function of position can achieve very high CNR for a range of anatomical placements.

Another consideration is whether the nonlinearity in the gradient affects the calculated ADC value. In the present design, nonlinearity over a $1 \mathrm{~mm}$ voxel with a median gradient of $500 \mathrm{mT} / \mathrm{m}$ contains deviations of approximately $\pm 10 \mathrm{mT} / \mathrm{m}$ or $2 \%$, but the effects of this deviation partially cancel across the voxel ${ }^{46}$. This amount of gradient variation across a voxel is comparable to that present in our experimental work, which achieved good agreement using just the midpoint gradient value for the b-value of each voxel. However, more realistic assignments of local b-value may show better performance.

In addition, the dependence of ADC values on TE, diffusion time and b-value is an active area of research, and several models have been proposed. We have tried to address some 
of these concerns by using a two-component model, which does impart b-value dependence to the measured ADC. However, this cannot be entirely resolved with simulations, particularly because the models are typically based on measurements with conventional hardware that cannot access the short diffusion times achieved here. However, this type of device could play an important role in further developing models that accurately characterize the microstructure that contributes to DWI contrast ${ }^{21-24}$.

For greater nonlinearity or higher-precision applications, it is also possible to derive the diffusion equations for a nonlinear gradient. We have performed this analysis for the simplest version of diffusion weighting, included in the supplementary material, which shows that nonlinearity can lead to similarly small deviations in the calculated ADCs $(\mathrm{O}(1 \%))$ that depend on the ratio of linear and nonlinear field contributions in the field across a voxel. For clinical applications, however, these corrections are never expected to be necessary. A greater factor skewing ADC values is the dependence on encoding and echo times, which has been demonstrated in human studies. However, stronger gradients only extend the potential to characterize ADC as a function of time and TE. Encouragingly, clinical studies have already shown that shorter encoding times improve the ability to distinguish cancerous and benign prostate $^{21,22}$.

Like linear gradients, the proposed device could induce eddy currents during an echoplanar imaging (EPI) readout. In the current experiments, long-lasting eddy currents were not observed, and images were reconstructed via FFT without additional corrections. Furthermore, the shorter echo times afforded by stronger gradients could potentially reduce the distortions associated with EPI. However, more complicated and persistent fields could be characterized via 2D phase-encoded mapping strategies, like those used to map dynamic nonlinear gradient encodings ${ }^{27,47,48}$. Once known, linear and certain nonlinear components could be physically compensated by appropriate waveforms, particularly since they need only be compensated over the small ROI of the prostate and also because diffusion gradient waveforms do not generally need to be changed with standard protocol changes. Finally, since even standard linear gradients are not truly linear, modern eddy correction post-processing techniques already model the observed eddy currents with a nonlinear basis ${ }^{57}$. With a change of basis, these same methods could be applied to the nonlinear gradient presented here.

Finally, while mechanical and thermal safety concerns were discussed in the Results, one 
important safety concern may be the potential for peripheral nerve stimulation. Achievable gradient moments in clinical imaging are often restricted by peripheral nerve stimulation (PNS) limits on the slew rate, but those limits are based on studies with full body gradients. More recent studies of PNS show that the overlap of anatomy with induced currents is the real key to PNS stimulation, and due to their highly localized nature, nonlinear gradients have long been hypothesized to produce less PNS for a given $\mathrm{dB} / \mathrm{dt}^{26,58}$. Furthermore, for this particular design, the most serious potential outcomes of PNS (i.e. stimulation of currents across the heart) are avoided, since the field and gradient are very small at that distance.

While true PNS limits are complex phenomena and will require additional testing for confirmation, the geometry of this device also provides important new degrees of freedom for mitigating PNS. In addition to lengthening ramp times, PNS can be further mitigated by shifting device placement a few centimeters.

Fig. 9 in the supplementary material shows $\mathrm{dB} / \mathrm{dt}$ assuming a $1 \mathrm{~ms}$ ramp time for a device placed $6 \mathrm{~cm}$ inferior to the perineum. With this geometry, only a small volume of the anatomy is subject to $\mathrm{dB} / \mathrm{dt}$ greater than $20 \mathrm{mT} / \mathrm{m}$ and, as shown in Fig. 5, CNR is still approximately double of that achieved with standard linear gradients ${ }^{59,60}$. 
E. H. Bhuiyan et al.

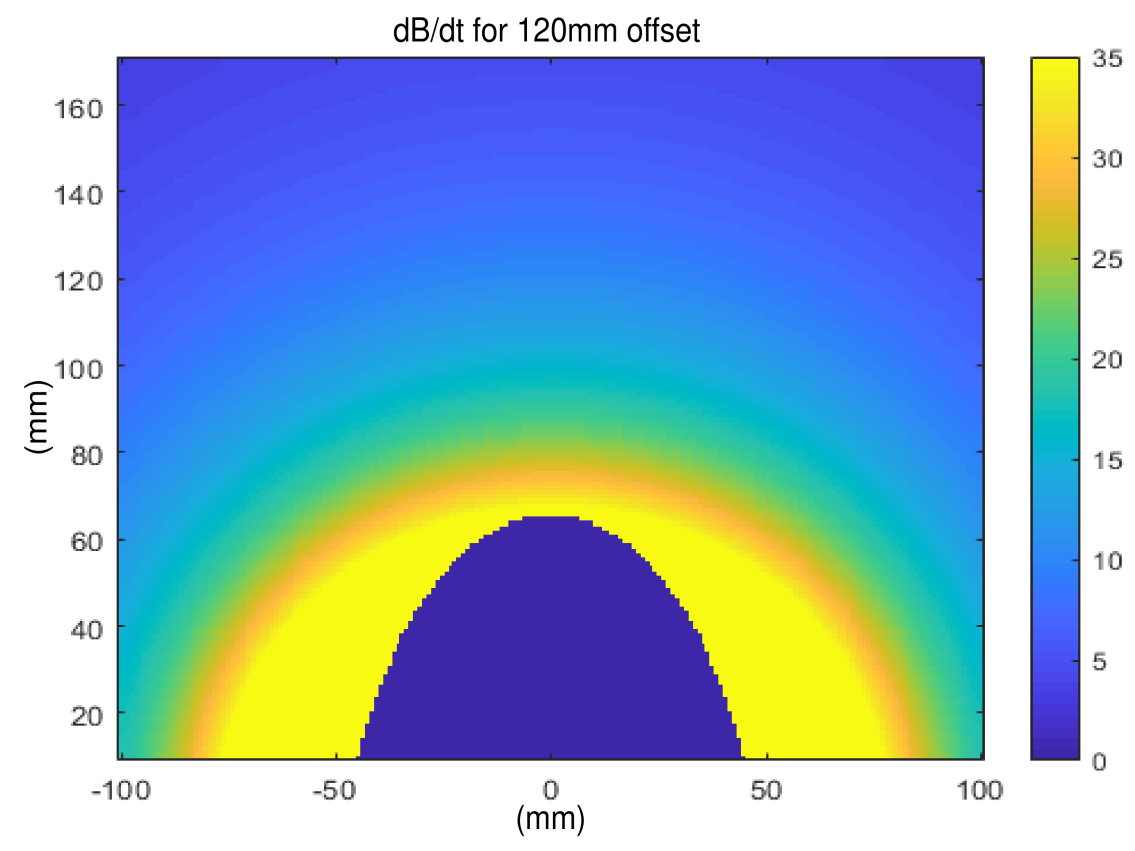

Figure 9: $\mathrm{dB} / \mathrm{dt}$ for nonlinear gradient. Peripheral nerve stimulation is a complicated phenomenon which is made more difficult to predict in the presence of nonlinear gradients. However, one advantage of the hardware proposed here is that PNS can be mitigated by device placement. This plot shows $\mathrm{dB} / \mathrm{dt}$ for a $1 \mathrm{~ms}$ ramp time with an offset of $120 \mathrm{~mm}$ from the prostate. The standard $20 \mathrm{~T} / \mathrm{s}$ slew is exceeded over a very small volume of the anatomy, reaching a maximum of $43 \mathrm{~T} / \mathrm{s}$ along the $\mathrm{x}=0$ line, with reductions in leg stimulation achievable by spacers or positioning. Notably, simulations for this positioning (Fig. 5) show that CNR is still approximately twice what is achievable with linear gradients. 


\section{Conclusion}

We present a new approach to improving DWI of prostate, introducing a new type of MRI accessory that delivers high diffusion weighting at short echo time, resulting in much stronger gradients and higher SNR. Prostate is anatomically ideal for diffusion weighting via a nonlinear inside-out gradient, and the clinical significance and increasing ubiquity of prostate MRI is likely to justify the additional hardware, which could be shared across many scanners. Our results predict a doubling of CNR, which could improve the reliability of MRI for prostate cancer imaging, which in turn could reduce both the high mortality and overtreatment rates for this widespread disease. 


\section{Appendix}

\section{VI.A. Theory}

The simplest calculation of diffusion weighting is to consider two infinitely short gradient pulses separated by a delay $\tau$. To simplify notation and without loss of generality, we consider spin with initial location $x(t=0)=\phi(t=0)=0$, so that $\Delta x=x$ and $\Delta \phi=\phi_{0}$. Over the delay, the spins have localized to a Gaussian distribution of positions,

$$
P(x)=e^{-\frac{x^{2}}{\sigma^{2}}}
$$

where $\sigma$ is related to the rms distance travel in time $\tau$. For linear gradients, $\mathrm{G}_{l}$, after the refocusing gradient lobe, the phase of each spin depends on its positions at $t=\tau$, $\phi(t=\tau, x) \equiv \phi(x)=k_{l} \tau x=k_{l} x$ and $d \phi=k_{l} d x$. We can therefore calculate the ensemble average of magnetization $M$. Assuming uniform spin density, the ensemble average of $M$ is proportional to the ensemble average of $e^{i \phi}$, which can be reformulated as an integral over $x$, as follows.

$$
\left\langle e^{i \phi}\right\rangle \propto \int_{-\infty}^{\infty} P(\phi) e^{i \phi} d \phi=\int_{-\infty}^{\infty} e^{-\frac{x^{2}}{\sigma^{2}}} e^{i k_{l} x} k_{l} d x=k_{l} \sqrt{\pi} \sigma e^{-\frac{k_{l}^{2} \sigma^{2}}{4}}
$$

The product $\sigma^{2} k_{l}^{2}$ reflects gradient winding, diffusion time, and diffusion rate. Thus the exponent is analogous to the product $b \star \mathrm{ADC}$ in the well-known Stejskal-Tanner equation ${ }^{18}$. Using the same simplified approach for a spin in the presence of both linear and a second order nonlinear field, the difference is in the relationship between $\phi$ and $x: \phi(t=\tau, x) \equiv$ $\phi(x)=G_{l} \tau x+G_{n l} \tau x^{2}=k_{l} x+k_{n l} x^{2}$ and $d \phi=\left(k_{l}+2 k_{n l} x\right) d x$ Now the integral to be evaluated is,

$$
\left\langle e^{i \phi}\right\rangle \propto \int_{-\infty}^{\infty} P(\phi) e^{i \phi} d \phi=\int_{\infty}^{-\infty} e^{\frac{-x^{2}}{\sigma^{2}}} e^{i\left(k_{l} x+k_{n l} x^{2}\right)}\left(k_{l}+2 k_{n l} x\right) d x
$$

Using standard formulas, we obtain

$$
\left\langle e^{i \phi}\right\rangle=k_{l}\left(\frac{\sqrt{\pi} \sigma}{\sqrt{1-\sigma^{2} k_{n l}}} e^{-\frac{k_{l}^{2} \sigma^{2}}{4\left(1-i \sigma^{2} k_{n l}\right)}}\right)+2 k_{n l}\left(\frac{\sqrt{\pi} i k_{l}}{2}\left(\frac{\sigma^{2}}{\left(1-i \sigma^{2} k_{n l}\right)}\right)^{3 / 2}\right) e^{-\frac{k_{l}^{2} \sigma^{2}}{4\left(1-i \sigma^{2} k_{n l}\right)}} .
$$


Rearranging, one can obtain:

$$
\left\langle e^{i \phi}\right\rangle=k_{l} \sqrt{\pi} \sigma e^{-\frac{\sigma^{2} k_{l}}{4\left(1-i \sigma^{2} k_{n l}\right)}}\left(\frac{1}{\sqrt{\left(1-i \sigma^{2} k_{n l}\right)}}+\frac{i k_{n l} \sigma^{2}}{\left(1-i \sigma^{2} k_{n l}\right)^{3 / 2}}\right) .
$$

Simplifying and removing complex values from the denominators, this becomes:

$$
\left\langle e^{i \phi}\right\rangle=k_{l} \sqrt{\pi} \sigma e^{-\frac{k_{l}^{2} \sigma^{2}}{4\left(1+\sigma^{4} k_{n l}^{2}\right)}} e^{-\frac{i \sigma^{4} k_{n l} k_{l}^{2}}{4\left(1+\sigma^{4} k_{n l}^{2}\right)}}\left(\frac{\sqrt{1+i \sigma^{2} k_{n l}}}{\sqrt{1+\sigma^{4} k_{n l}^{2}}}+\frac{i k_{n l} \sigma^{2}\left(1+i \sigma^{2} k_{n l}\right)^{3 / 2}}{\left(1+\sigma^{4} k_{n l}^{2}\right)^{3 / 2}}\right)
$$

As expected, when $k_{n l}$ is zero this matches the linear gradient expression. More, generally, neglecting nonlinear winding will cause a slight underestimation of the effective diffusion weight, which will lead to a slight overestimation of ADCs by a scaling factor of $\left(1+\sigma^{4} k_{n l}^{2}\right)$. This could bias ADCs by a few percent, if not corrected. The imaginary term in the exponent indicates that nonlinear winding can also add a periodic phase shift in the signal, similar to what is observed with dephasing under a nonlinear gradient ${ }^{41}$.

The complex factor in braces can alter both the magnitude and phase of the signal, in principle. However, in practice $\sigma \approx 10^{-2} \mathrm{~cm}$ and $k_{n l} \approx 10^{2}-10^{3} \mathrm{~Hz} / \mathrm{cm}^{2}$, over which the magnitude of this term ranges from 1 to 0.993 and its phase changes by less than 9 degrees. These corrections are highly unlikely to have clinical significance even in high precision work.

\section{Acknowledgment}

The authors gratefully acknowledge the efforts of Xavier Mooney, Ben Leigh, and Steve Bates at Tesla Engineering Ltd., for providing initial design specifications. Advice by Terry Nixon of the Yale Magnetic Resonance Research Center was also extremely helpful and is greatly appreciated. 


\section{References}

1 Key Statistics for Prostate Cancer. American Cancer Society. https://www.cancer.org/cancer/prostate-cancer/detection-diagnosis-staging/acsrecommendations.html. Accessed 2020.

2 Brawley OW. Trends in Prostate Cancer in the United States. J Natl Cancer Inst Monogr. 2012;45:152-156. doi:10.1093/jncimonographs/lgs035.

3 Rawla P. Epidemiology of Prostate Cancer. World J Oncol. 2019;10(2):63-89. https://doi.org/10.14740/wjon1191.

4 Klotz L, "Prostate cancer overdiagnosis and overtreatment. Curr Opin Endocrinol Diabetes Obes.2013;20(3):204-209. doi:10.1097/MED.0b013e328360332a.

5 Loeb S, Bjurlin MA, Nicholson J, et al. Overdiagnosis and Overtreatment of prostate cancer. Eur Urol. 2014;65(6):1046-1055. doi:10.1016/j.eururo.2013.12.062.

6 Ahmed HU, El-Shater AB, Brown LC, et al. Diagnostic accuracy of multi-parametric MRI and TRUS biopsy in prostate cancer (PROMIS): a paired validating confirmatory study. Lancet 2017:389(10071):815-822. doi:10.1016/S0140-6736(16)32401-1.

7 Algohary A, Viswanath S, Shiradkar R, et al. Radiomic Features on MRI Enable Risk Categorization of Prostate Cancer Patients on Active Surveillance: Preliminary Findings. J. Magn. Reson. Imaging 2018;48(3):818-828. doi: 10.1002/jmri.25983.

8 Weinreb JC, Barentszb JO, Choykec PL, et al. PI-RADS Prostate ImagingReporting and Data System: 2015. Version 2. Eur Urol. 2016;69(1):16-40. doi:10.1016/j.eururo.2015.08.052.

9 Padhani AR, Liu G, Koh DM, et al. Diffusion-Weighted Magnetic Resonance Imaging as a Cancer Biomarker: Consensus and Recommendations. Neoplasia 2009;11(2):102-125. doi:10.1593/neo.81328.

10 deSouza NM, Riches SF, Vanas NJ, et al. Diffusion-weighted magnetic resonance imaging: a potential non-invasive marker of tumour aggressiveness in localized prostate cancer. Clin Radiol. 2008;63(7):774-782. doi:10.1016/j.crad.2008.02.001. 
11 van Leeuwen PJ, Hayen A, Thompson JE, et al. A multiparametric magnetic resonance imaging-based risk model to determine the risk of significant prostate cancer prior to biopsy, BJU Int, 2017;120(6):774-481. doi: 10.1111/bju.13814.

12 Johnson LM, Turkbey B, Figg WD, et al. Multiparametric MRI in prostate cancer management. Nat Rev Clin Oncol. 2014;11(6):346-353. doi:10.1038/nrclinonc.2014.69.

13 Mehralivand S, Shih JH, S. Rais-Bahrami S, et al. A Magnetic Resonance Imaging-Based Prediction Model for Prostate Biopsy Risk Stratification. JAMA Oncol.;4(5):678-685. doi:10.1001/jamaoncol.2017.5667.

14 Kasivisvanathan V, Antti SR, Borghi M, et al. MRI-Targeted or Standard Biopsy for Prostate-Cancer Diagnosis. $N$ Engl J Med. 2018;378(19):1767-1777. doi:10.1056/NEJMoa1801993.

15 Siddiqui MM, Rais-Bahrami S, Turkbey B, et al. Comparison of MR/ultrasound fusionguided biopsy with ultrasound-guided biopsy for the diagnosis of prostate cancer. JAMA. 2015;313(4):390-397. doi:10.1001/jama.2014.17942.

16 Muller BG, Shih JH, Sankineni S, et al. Prostate cancer: interobserver agreement and accuracy with the revised Prostate Imaging Reporting and Data System at multiparametric MR imaging. Radiology 2015;277(3):741-750. doi:10.1148/radiol.2015142818.

17 Callaghan PT. Structural Imaging Using q-Space. Principles of Nuclear Magnetic Resonance Microscopy. New York: Oxford University Press Inc.; 1991. 400-405 p.

18 Stejskal EO, Tanner JE, "Spin diffusion measurements: Spin echoes in the presence of a time-dependent field gradient. J Chem Phys. 2004;42(1):288-292. https://doi.org/10.1063/1.1695690.

19 Rosenkrantz AB, Chandarana H, Hindman N, et al. Computed diffusion-weighted imaging of the prostate at $3 \mathrm{~T}$ : impact on image quality and tumour detection. Eur Radiol. 2013;23:3170-3177. doi:10.3348/kjr.2018.19.5.832

20 Chatterjee A, Watson G, Myint E, et al. Changes in Epithelium, Stroma, and Lumen Space Correlate More Strongly with Gleason Pattern and Are Stronger Predictors 
of Prostate ADC Changes than Cellularity Metrics. Radiology. 2015;277(3):751-762. doi:10.1148/radiol.2015142414.

21 Lemberskiy G, Rosenkrantz AB, Veraart J, et al. Time-Dependent Diffusion in Prostate Cancer.Investigative Radiology 2017;52(7):405-411. doi:10.1097/RLI.0000000000000356.

Lemberskiy G, Fieremans E, Veraart J, et al. Characterization of Prostate Microstructure Using Water Diffusion and NMR Relaxation. Front. Phys. 2018;6:91. https://doi.org/10.3389/fphy.2018.00091.

Gilani N, Malcolm P, and Johnson G. A Model Describing Diffusion in Prostate Cancer. Magn Reson Med. 2016;78(1):316-326. doi:10.1002/mrm.26340.

Panagiotaki E, Chan RW, Dikaios N, et al. Microstructural Characterization of Normal and Malignant Human Prostate Tissue With Vascular, Extracellular, and Restricted Diffusion for Cytometry in Tumours Magnetic Resonance Imaging. Invest Radiol. 2015;50(4):218-227. doi:10.1097/RLI.0000000000000115.

Ito S, Yamada Y. Alias-free image reconstruction using Fresnel transform in the phase-scrambling Fourier imaging technique. Magn Reson Med. 2008;60(2):422-430. doi:10.1002/mrm.21672.

26 Hennig J, Welz AM, Schultz G, et al. Parallel imaging in non-bijective, curvilinear magnetic field gradients: a concept study. Magnetic Resonance Materials in Physics, Biology and Medicine 2008;21(1):5-14. doi:10.1007/s10334-008-0105-7.

Stockmann JP, Galiana G, Tam L, et al. In vivo O-Space imaging with a dedicated 12 cm Z2 insert coil on a human 3T scanner using phase map calibration. Magn Reson Med. 2013;69(2):444-455. doi:10.1002/mrm.24282.

28 Galiana G, Stockmann JP, Tam L, et al. The role of nonlinear gradients in parallel imaging: A kspace based analysis. Concepts in Magnetic Resonance Part A. 2012;40A(5):253267. doi:10.1002/cmr.a.21243.

Hidalgo-Tobon SS, Theory of Gradient Coil Design Methods for Magnetic Resonance Imaging. Concepts in Magnetic Resonance Part A 2010;36A(4):223-242. doi:10.1002/cmr.a.20163. 
30 Setsompop K, Kimmlingen R, Eberlein E, et al. Pushing the limits of in vivo diffusion MRI for the Human Connectome Project. Neuroimage. 2013;80:220-233. doi:10.1016/j.neuroimage.2013.05.078.

31 Murphy G, Haider M, Ghai S, et al. The expanding role of MRI in prostate cancer. AJR Am J Roentgenol. 2013;201(6):1229-1238. doi:10.2214/AJR.12.10178.

32 Hadaschik B. [Paradigm shift in urology: Prostate cancer diagnosis using MRI-targeted or standard transrectal ultrasonography-guided biopsy]. Urologe A. 2018;57(6):727728. doi:10.1007/s00120-018-0668-5.

33 Merrett C, Mannas M, Black PC, et al. Magnet Before the Needle Commentary on: MRI-targeted or Standard Biopsy for Prostate-cancer Diagnosis (PRECISION Trial). Urology 2018;118:1-2. doi:10.1016/j.urology.2018.04.024.

34 Mowatt G, Scotland G, Boachie C, et al. The diagnostic accuracy and cost-effectiveness of magnetic resonance spectroscopy and enhanced magnetic resonance imaging techniques in aiding the localisation of prostate abnormalities for biopsy: a systematic review and economic evaluation. Health Technology Assessment 2013;17(20):vii-xix, 1-281. doi:10.3310/hta17200.

35 de Rooij M, Crienen S, Witjes JA, et al. Cost-effectiveness of magnetic resonance (MR) imaging and MR-guided targeted biopsy versus systematic transrectal ultrasound-guided biopsy in diagnosing prostate cancer: a modelling study from a health care perspective. Eur Urol. 2013;66(3):430-436. doi:10.1016/j.eururo.2013.12.012.

36 R. Renard-Penna R, O. Rouvire O, P. Puech P, et al. Current practice and access to prostate MR imaging in France. Diagn Interv Imaging 2016;97(11):1125-1129. doi:10.1016/j.diii.2016.06.010.

37 Venderink W, Govers TM, de Rooij M, et al. Cost-Effectiveness Comparison of Imaging-Guided Prostate Biopsy Techniques: Systematic Transrectal Ultrasound, Direct In-Bore MRI, and Image Fusion. AJR Am J Roentgenol. 2017;208(5):1058-1063. doi:10.2214/AJR.16.17322. 
Mueller-Lisse UG, Murer S Mueller-Lisse UL, et al. Everymans prostate phantom: kiwifruit substitute for human prostates at magnetic resonance imaging, diffusion-weighted imaging and magnetic resonance spectroscopy. Eur Radiol 2017;27(8):3362-3371.

Jendoubi S, Wagner M, Montagne S, et al. MRI for prostate cancer: can computed high b-value DWI replace native acquisitions? European Radiology 2019;29:5197-5204. https://doi.org/10.1007/s00330-019-06085-z.

High Precision Devices, Inc., 4601 Nautilus Ct S Boulder, CO 80301 USA. https://doi.org/10.1007/s00330-016-4706-7.

41 Wagner F, Laun FB, Kuder TA, Mlynarska A, Maier F, Faust J, et al. Temperature and concentration calibration of aqueous polyvinylpyrrolidone (PVP) solutions for isotropic diffusion MRI phantoms. PLoS ONE 2017;12(6). https://doi.org/10.1371/journal.

Jensen JH, Helpern JA, Ramani A, et al. Diffusional kurtosis imaging: the quantification of non-Gaussian water diffusion by means of magnetic resonance imaging. Magn Reson Med. 2005;53(6):14321440. doi.org/10.1002/mrm.20508.

Si Y, Liu RB. Diagnostic Performance of Monoexponential DWI Versus Diffusion Kurtosis Imaging in Prostate Cancer: A Systematic Review and Meta-Analysis. AJR Am J Roentgenol. 2018;211(2):358-368. doi:10.2214/AJR.17.18934.

Stockmann JP, Ciris PA, Galiana G, et al. OSpace imaging: Highly efficient parallel imaging using secondorder nonlinear fields as encoding gradients with no phase encoding. Magn Reson Med. 2010;64(2):447-456. doi:10.1002/mrm.22425.

45 Tam LK, Stockmann JP, Galiana G, et al. Null Space Imaging: Nonlinear Magnetic Encoding Fields Designed Complementary to Receiver Coil Sensitivities for Improved Acceleration in Parallel Imaging. Magn Reson Med. 2011;68(4):1166-1175. doi:10.1002/mrm.24114. implications for imaging and field mapping. Magn Reson Med. 2012;67(4):1120-1126. doi:10.1002/mrm.23085. 
47 Wang H, Tam LK, Constable RT, et al. Fast rotary nonlinear spatial acquisition (FRONSAC) imaging. Magn Reson Med. 2016;75(3):1154-1165. doi:10.1002/mrm.25703.

48 Dispenza NL, Littin S, Zaitsev M, et al. Clinical Imaging Potential of FRONSAC. Nature Scientific Reports 2019;9(1):1912. doi:10.1038/s41598-018-36802-5.

49 Littin S, Feng Jia F, Amrein P, et al. Single channel non-linear breast gradient coil for diffusion encoding. Proc. Intl. Soc. Mag. Reson. Med. 28 (2020).

50 Wetter A, Nensa F, Lipponer C, et al. High and ultra-high b-value diffusion-weighted imaging in prostate cancer: a quantitative analysis. Acta Radiol. 2018;56(8):10091010doi:10.1177/0284185114547900.

51 Woodfield CA, Tung GA, Grand DJ, et al. Diffusion-Weighted MRI of Peripheral Zone Prostate Cancer: Comparison of Tumor Apparent Diffusion Coefficient With Gleason Score and Percentage of Tumor on Core Biopsy. AJR Am J Roentgenol. 2010;194(4):W316-W322. doi:10.2214/AJR.09.2651.

52 Hurrell SL, McGarry SD, Kaczmarowski A, et al. Optimized b-value selection for the discrimination of prostate cancer grades, including the cribriform pattern, using diffusion weighted imaging. J. Med. Imag. 2017;5(1):011004. doi:10.1117/1.JMI.5.1.011004.

53 Rosenkrantz AB, Parikh N, Kierans AS, et al. Prostate Cancer Detection Using Computed Very High b-value Diffusion-weighted Imaging: How High Should We Go? Acad Radiol. 2016;23(6):704-711. doi:10.1016/j.acra.2016.02.003.

54 Ueno Y, Takahashi S, Ohno Y, et al. Computed diffusion-weighted MRI for prostate cancer detection: the influence of the combinations of b-values. Br J Radiol. 2015;88(1048): 20140738. doi: 10.1259/bjr.20140738 [online].

55 Metens T, Miranda D, Absil J, et al. What is the optimal b value in diffusionweighted MR imaging to depict prostate cancer at 3T?. Eur Radiol 2012;22:703709. https://doi.org/10.1007/s00330-011-2298-9.

56 Liss MA, White NS, Parsons JK, et al. MRI-Derived Restriction Spectrum Imaging Cellularity Index is Associated with High Grade Prostate Cancer on Radical Prostatectomy Specimens. Front Oncol. 2015;5(30). doi:10.3389/fonc.2015.00030. 
E. H. Bhuiyan et al.

57 Andersson JLR, Sotiropoulos SN. An integrated approach to correction for off-resonance effects and subject movement in diffusion MR imaging. NeuroImage. 2016;15(125):10631078. doi:10.1016/j.neuroimage.2015.10.019.

58 Davids M, Gurin B, Malzacher M, Schad LR, Wald LL. Predicting Magnetostimulation Thresholds in the Peripheral Nervous System Usign Realistic Body Models. Scientific Reports. 2017;7(1):5316. doi:10.1038/s41598-017-05493-9.

59 A Primer on Medical Device Interactions with Magnetic Resonance Imaging Systems. wwfdagov/MedicalDevices/DeviceRegulationandGuidance/GuidanceDocuments/ucm10r721htm. Accessed 2009.

60 Submission of Premarket Notifications for Magnetic Resonance Diagnostic Devices: Guidance for Industry and Food and Drug Administration Staff. In: FDA DoRH, ed. 2016. 